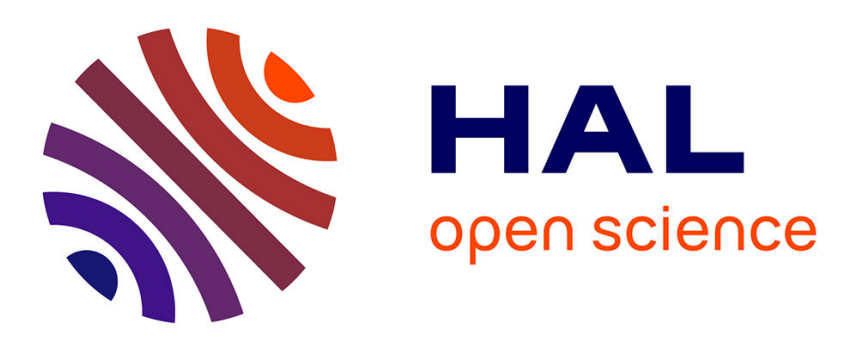

\title{
Impact économique de la construction de la LGV SEA Tours-Bordeaux sur les régions traversées \\ Etienne Fouqueray
}

\section{To cite this version:}

Etienne Fouqueray. Impact économique de la construction de la LGV SEA Tours-Bordeaux sur les régions traversées. Revue d'économie régionale et urbaine, 2016, Mars (2), pp.385-416. 10.3917/reru.162.0385. hal-00996965v2

\section{HAL Id: hal-00996965 \\ https://hal.science/hal-00996965v2}

Submitted on 14 Oct 2016

HAL is a multi-disciplinary open access archive for the deposit and dissemination of scientific research documents, whether they are published or not. The documents may come from teaching and research institutions in France or abroad, or from public or private research centers.
L'archive ouverte pluridisciplinaire HAL, est destinée au dépôt et à la diffusion de documents scientifiques de niveau recherche, publiés ou non, émanant des établissements d'enseignement et de recherche français ou étrangers, des laboratoires publics ou privés.

$$
\text { Copyright }
$$




\title{
IMPACT ECONOMIQUE DE LA CONSTRUCTION DE LA LGV SEA TOURS-BORDEAUX SUR LES REGIONS TRAVERSEES ${ }^{1}$
}

\author{
Fouqueray, Etienne \\ CRIEF EA 2249 - Université de Poitiers
}

Version provisoire 2

Les propos tenus dans cette publication n'engagent que leur auteur.

\begin{abstract}
Résumé
Du point de vue de la théorie de la croissance régionale, les chantiers de grandes infrastructures de transport sont souvent perçus comme un moyen de favoriser le développement économique des territoires traversés à court terme. Dans un contexte où la contribution des acteurs locaux au financement des nouvelles infrastructures représente un enjeu considérable, l'évaluation des retombées économiques locales des grands chantiers devient une nécessité technique et politique de plus en plus forte. A partir d'un rappel des fondements de la théorie keynésienne, nous proposons dans cet article une application d'un modèle d'impact économique appliqué au cas de la construction de la Ligne à Grande Vitesse (LGV) Sud Europe Atlantique (SEA) Tours-Bordeaux. Bien que non suffisante pour légitimer l'investissement conséquent réalisé dans ce projet, cette modélisation est un préalable à un calcul plus vaste de rentabilité sociale du projet SEA. A partir d'un modèle Input-Output hybride s'appuyant sur un travail d'enquête conséquent et sur des données nationales de l'INSEE, nous évaluons l'impact économique de cette construction sur une période de deux ans (2011-2013) pour l'ensemble des régions Aquitaine, Centre et Poitou-Charentes. II apparaît qu'un euro dépensé localement dans la construction augmente de 1,96 euros la production dans ces régions. Au total, 13799 emplois, 1,6 milliards d'euros de production et 755 millions d'euros de valeur ajoutée ont été générés.
\end{abstract}

\section{Mots-clés}

Infrastructure de transport, Construction, Impact local, Modélisation, Base économique, Input-Output, Théorie keynésienne.

\footnotetext{
${ }^{1}$ Cette recherche n'aurait pas été ce qu'elle est sans les conseils avisés d'Olivier Bouba-Olga, de Dominique Vollet et de Jean-Jacques Gouguet. Je tiens à les remercier pour leur disponibilité.
} 


\section{Introduction}

La Ligne à Grande Vitesse (LGV) Sud Europe Atlantique (SEA) entre Tours et Bordeaux est la première LGV française construite et exploitée sous la forme d'une concession. Par ce mode de financement, Réseau Ferré de France et de l'Etat réduisent leur investissement et le risque y étant associé en le faisant supporter par le secteur privé mais aussi par les collectivités territoriales traversées par la ligne. Le coût de la construction, intégrant également l'activité du concessionnaire durant la période de travaux, avoisine les 6,2 milliards d'euros. La contribution des collectivités territoriales des trois régions traversées représente un peu plus d'un milliard d'euros. Dans ce contexte, le chantier le plus important d'Europe suscite des attentes fortes en termes de retombées économiques. Débutée en 2011, la construction de la LGV SEA Tours-Bordeaux s'achèvera en 2016 pour une mise en service programmée en 2017. Dans cet article, nous proposons de modéliser et d'estimer à mi-parcours (mars 2013) l'impact économique des dépenses effectuées pour la construction de la ligne dans les trois régions traversées. A cette date, l'avancement du projet était de 41,5\%.

Les modèles d'impact économique s'appuient sur le corpus théorique de la croissance régionale et plus précisément sur la théorie de la base économique développée dans la première moitié du XXe siècle (Hoyt, 1954; North, 1955; Sombart, 1902; Tiebout, 1956) et revisité plus récemment par Davezies (2008). Ils cherchent à « quantifier les conséquences économiques d'une activité permanente (une base militaire, un équipement sportif, etc.) ou temporaire (un festival, un évènement sportif, etc.) sur un territoire déterminé » (Barget et Gouguet, 2011). Alors qu'à l'échelle d'un Etat, la croissance est fortement impactée par l'investissement, la consommation, l'épargne, et d'autres agrégats économiques, au niveau d'une région, il apparaît que le développement économique dépend de la demande extérieure (Vollet et Brétière, 2008) et de la capacité du territoire à capter des revenus qui lui sont extérieurs (revenus basiques). Le secteur basique va développer le secteur non basique en fonction de la propension à dépenser localement des agents économiques (entreprises, consommateurs, administrations publiques). L'accroissement de l'emploi dans les secteurs basiques et non basiques générés par l'injection de revenus extérieurs engendrera à son tour une croissance du revenu (McCann, 2001). L'effet multiplicateur sera alors d'autant plus important que les fuites de revenus à l'extérieur de la région seront limitées (Vollet et Dion, 2001).

Les modèles d'impact économique permettent donc d'étudier la manière dont la demande de biens et services se répartit dans l'économie locale suite à un choc sur un secteur. Ils ne s'intéressent qu'aux bénéfices générés par les projets, sans prendre en compte tous les coûts (congestion, effets d'éviction, formation professionnelle, etc.) qui les accompagnent, ce qui tend à créer un biais en faveur des projets très coûteux (Barget et Gouguet, 2011; Stringer et al., 1992). De fait, l'impact économique à lui seul ne suffit pas pour légitimer le financement d'un projet, ni pour en évaluer l'ensemble des effets pour les territoires et leurs habitants (Barget et Gouguet, 2011). Ainsi, notre évaluation d'impact ne représente qu'une partie des effets observables de la construction de la LGV SEA entre Tours et Bordeaux. Elle nécessite des investigations complémentaires pour se prononcer sur la rentabilité sociale du projet. Par ailleurs, il s'agit d'une démarche court-termiste qui ne permet pas de se prononcer sur l'effet de long terme de la construction de la LGV SEA Tours-Bordeaux dans les régions traversées.

Dans une première partie, nous soulignons la nécessité de mener des évaluations sur l'impact économique des constructions d'infrastructures de transports pour les territoires traversés. Nous décrivons alors les limites des pratiques d'évaluation de l'impact économique des grands chantiers en France et posons le cadre théorique dans lequel nous nous inscrivons. Après avoir synthétisé les fondements de la théorie keynésienne concernant l'effet multiplicateur de programmes de relance via l'investissement dans de grands projets, nous rappelons les différents modèles d'impact économique existants et explicitons notre choix de modélisation. Dans une seconde partie, nous décrivons les différents termes du modèle d'impact que nous retenons ainsi que les données que nous mobilisons. Nous montrons que la spécificité du projet évalué (part élevée des salariés en déplacement et des fournisseurs extérieurs aux régions traversées, présence éphémère du constructeur sur le territoire) nécessite de construire un modèle hybride mêlant enquêtes de terrain et collectes de données existantes. Dans la dernière partie, nous proposons plusieurs estimations de l'impact économique en fonction des hypothèses retenues. Après avoir constaté que les quatre estimations les plus robustes aboutissaient à des résultats proches, nous retenons une moyenne de ces estimations pour évaluer l'impact économique de la construction de la LGV SEA. Sur la période étudiée, nous montrons que celle-ci a généré dans les trois régions traversées 13799 emplois, 1,6 milliards d'euros de production et 755 millions d'euros de valeur ajoutée. L'effet multiplicateur sur la production de l'injection nette dans la construction est de 1,96. Les retombées économiques se 
concentrent prioritairement dans les secteurs de la construction, de la fabrication d'autres produits industriels, des industries extractives, des activités scientifiques et techniques, et secondairement dans ceux de l'agriculture, de la fabrication de denrées alimentaires, de l'hébergement et de la restauration, et du commerce.

\title{
1. L'évaluation de l'impact économique des constructions de grandes infrastructures de transport: enjeux, fondements et instruments
}

\author{
1.1. La construction des grandes infrastructures: un enjeu d'évaluation de l'impact économique \\ régional
}

Souvent considérée comme une activité non délocalisable par nature et à forte intensité de main d'œuvre (OCDE, 2002), la construction des grandes infrastructures de transport suscite de fortes attentes en termes de retombées économiques. Si à l'échelle nationale les richesses générées par la construction d'une grande infrastructure sont diffuses et quasiment imperceptibles, à l'échelle régionale elles peuvent être non négligeables (Berion, 2002; Berion et al., 2005; Burmeister, 1997; Petitjean, 2003). Conscient de l'enjeu pour les territoires traversés d'accueillir des grands chantiers d'infrastructure pour favoriser leur développement économique à court terme, les financeurs publics et privés de tels projets n'hésitent pas à solliciter leur soutien financier. Parties prenantes, ces acteurs locaux exigent en retour des retombées économiques perceptibles et évaluables. La construction de la LGV SEA Tours-Bordeaux n'échappe pas à ce contexte et à cette demande sociale. Une enquête menée par l'Observatoire socio-économique de la ligne en 2012 a révélé que la question des retombées économiques représentait l'attente prioritaire des acteurs locaux (Manceau et al., 2012).

Dans le cadre de grands chantiers d'infrastructures de transport, les évaluations peuvent prendre plusieurs formes et chercher à répondre à différentes interrogations. En amont de la finalité ultime de ces évaluations qui est d'estimer la rentabilité sociale des projets (Barget et Gouguet, 2010), d'autres questions peuvent être investiguées. Une question récurrente et souvent étudiée par les chercheurs est celle de l'impact économique d'une construction et de son effet multiplicateur sur l'économie locale. Bien que les méthodes de modélisation de l'impact économique des chantiers de grandes infrastructures de transport sur les territoires soient connues depuis longtemps par les économistes (Cohen et al., 2012; Lynch, 2000; OCDE, 2002; Taroux, 1988; Wubneh, 2008), les applications à des cas concrets ne sont pas systématisées, notamment en France. A défaut d'une véritable mesure, l'impact économique est alors souvent estimé à partir de multiplicateurs obtenus sur d'autres chantiers (Réseau Ferré de France, 2010), ce qui présente des biais liés aux particularités des projets évalués, des territoires dans lesquels ces investissements ont lieu et aux moments des constructions (Barget et Gouguet, 2011). Trois raisons principales expliquent bien souvent l'absence d'évaluation : i) le manque d'informations sur les coûts de la construction et leur géographie ii) la temporalité ex-post des évaluations accentuant de facto la perte d'informations, iii) l'absence de données régionales françaises sur les relations entre branches d'activité impliquant un travail d'enquête conséquent (Barget et Gouguet, 2011; Garrabé, 2008; Lynch, 2000; Wubneh, 2008). Le fait que notre recherche se fasse pendant le chantier nous permet de dépasser ces difficultés et d'estimer, à partir de données détaillées et d'enquêtes, l'impact économique de la construction de la LGV SEA Tours-Bordeaux.

\subsection{Les évaluations d'impact économique : fondements théoriques}

Lorsqu'on évalue l'impact économique d'un projet sur un territoire, on cherche à savoir si son financement va agir comme un choc de demande sur l'économie territoriale et avec quelle intensité. De ce point de vue, les méthodes d'évaluation d'impact économique s'inscrivent dans le cadre de la théorie keynésienne. Le financement d'un projet par la puissance publique, locale ou nationale, peut donc être considéré comme une politique de relance dont l'objectif est d'accroître la demande puis l'activité dans une logique de cercle vertueux. Ce type de programme trouve sa justification dans le fait que les entreprises, faute de détenir une information parfaite, ne parviennent pas seules à s'ajuster à la demande effective et donc au plein emploi. Pour faire face à cette situation et favoriser l'emploi en période de sous-emploi, la puissance publique peut donc choisir de financer 
l'activité économique sous diverses formes dont les grands projets d'infrastructure. Ainsi, l'investissement consenti est supposé avoir un effet multiplicateur sur l'activité économique. Autrement dit, un euro investit dans un projet génèrera plus de un euro de production dans l'économie territoriale. Dans le cadre d'une économie ouverte, les politiques de relance par la demande sont critiquées car elles alimentent par effets d'entraînement la croissance d'autres économies, tout en creusant les déficits publics de l'institution en étant à l'initiative. Toutefois, lorsqu'elles ciblent des activités très ancrées localement et peu ouvertes vers d'autres économies, ces politiques de relance peuvent avoir un réel impact économique pour les territoires concernés. L'intérêt des modèles d'impact économique est donc d'évaluer l'effet d'une politique de relance sur un territoire, en mesurant notamment son effet multiplicateur sur l'activité économique locale. En appliquant ce type d'évaluation à différents projets et politiques de relance, il est possible de comparer leurs efficacités respectives. Toutefois, plusieurs éléments limitent ces comparaisons. Ainsi, les notions d'horizon temporel et d'espace pertinent jouent un rôle important dans les résultats des modèles d'impact économique. Par exemple, pour un même type de projet, plus le territoire d'étude sera étendu et intégré économiquement, plus l'effet multiplicateur et les retombées induites seront élevés.

Par ailleurs le choix pour l'Etat de financer un projet sur un territoire implique que d'autres projets sur d'autres territoires ne pourront pas bénéficier de ce financement. En effet, le financement de grands projets dans l'optique d'une politique de relance est fortement contraint par les ressources des pouvoirs publics. Du fait de son caractère très localisé, la construction de la LGV SEA Tours-Bordeaux s'accompagne d'inégalités spatiales quant à ses effets, notamment observables par l'étude de la répartition spatiale des montants de sous-traitance et de fourniture (Fouqueray, 2013). D'un point de vue régional, la contribution des acteurs publics (Etat, collectivités territoriales) aurait pu être utilisée pour le financement d'autres projets qu'un projet de construction d'infrastructure de transport. N'étant pas en mesure d'évaluer ex ante le coût d'opportunité (Aandréani, 1967; Green, 1894) que représente ce choix de financement vis à vis d'autres projets possibles, nous ne pouvons pas nous prononcer sur la pertinence d'orienter l'argent public vers l'investissement dans la construction de la LGV SEA plutôt que vers d'autres projets locaux.

\subsection{L'évaluation d'impact de court-terme : concepts et instruments}

Les chercheurs distinguent trois grands types de modèles d'impact économique d'inspiration keynésienne (Inputoutput, keynésien, base économique) auxquels on peut ajouter un quatrième type de modèle plus sophistiqué mais aussi plus coûteux à mettre en œuvre : les modèles d'équilibre général (Blake, 2005) calibré généralement sur des matrices de comptabilité sociale (Decaluwé et al., 2001; Fofana, 2007). Ces derniers sont une « sophistication du modèle Input-Output» (Barget et Gouguet, 2011).

Aux Etats-Unis et dans les pays anglo-saxons, il existe une longue histoire de l'évaluation d'impact depuis les années 1950 liée à la volonté de mesurer l'effet de politiques industrielles sur le développement économique des régions. Les travaux précurseurs de North (1955) et Tiebout (1956), notamment, ont été poursuivi par de nombreux auteurs dont Richardson synthétise parfaitement les travaux (1985). Mathématiquement identiques lorsqu'ils sont correctement formulés, les modèles de la base économique et les modèles Input-Output se sont développés conjointement jusqu'au début des années 1980 (Richardson², 1985). Depuis la fin des années 1980, les modèles Input-Output (RIMSII IMPLAN et REMI) ont pris le pas sur les autres du fait notamment de l'existence de données régionales sur les relations entre branches d'activité permettant la construction de matrices de Léontief régionales. Au fil du temps, l'utilisation de ces matrices régionales s'est imposée comme une nécessité pour les économistes américains souhaitant réaliser des évaluations d'impact ( Lynch $\left.^{3}, 2000\right)$. Les derniers développements de ces modèles anglo-saxons très sophistiqués tendent à se concentrer dorénavant sur

\footnotetext{
2 « In any event, it has been repeatedly demonstrated [Billing 1969 ; Garnick, 1970, Isard and Czamanski, 1965 ; Romanoff, 1974] that, when appropriately defined, economic base and input-output models are mathematically identical ».

3 « [...] systematic analysis of economic impacts must account for the inter-industry relationships within regions because these relationships largely determine how regional economies are likely to respond to project an program changes. Thus, regional input-output (I-O) multipliers, which account for inter-industry relationships within regions, are useful tools for conducting economic impact analysis »
} 
des éléments de précision tels que la prise en compte de l'évolution de l'indice des prix à la consommation ou de la productivité.

En France, il n'existe pas de tableaux entrées-sorties au niveau régional. Pour combler ce manque, la plupart des études d'impact mobilisent des modèles de la base économique, moins couteux à développer que des modèles Input-Ouput et parfaitement appropriés pour des évaluations portant sur des territoires de petite taille faiblement intégrés (Vollet et Dion, 2001). Pour autant, il est possible d'utiliser des matrices de Léontief nationales pour peu que des données issues d'enquêtes permettent de les corriger et de tenir compte des spécificités locales (Catin et Nicolini, 2005). Dans les années 1980, sans présager du développement des données régionales américaines, Richardson soulignait déjà que l'avenir des modèles Input-Ouput consisterait à combiner des données issues d'enquêtes et des données issues de table " sans enquête " pour aboutir à un modèle hybride.

Bien souvent le choix d'un type de modèle dépend davantage des données disponibles et « des objectifs poursuivis par la modélisation» (Vollet, 2014) que d'un positionnement théorique. II n'existe pas une méthode standard qui serait applicable à tous les projets : " les travaux en matière sportive comme ailleurs étant particulièrement hétérogènes. Des angles d'observation divergents sont retenus d'une étude à une autre » (Barget et Gouguet, 2011). Conscient des avantages et des limites des différents modèles d'impact économique, et tenant compte des données dont nous disposons, nous avons retenus un modèle hybride qui associe enquêtes de terrain, données du constructeur et données nationales. Nous avons fait le choix de retenir comme territoire d'étude une grande région regroupant les trois régions administratives traversées par la LGV SEA. Ce découpage a le mérite de donner une vue globale de l'impact économique régional de la construction de la LGV SEA. Cependant, il ne permet pas de mettre en lumière les différences infrarégionales quant à sa diffusion. Or le caractère linéaire d'un projet de construction d'infrastructure limite bien souvent une part non négligeable des retombées économiques aux communes frontalières du tracé (Berion, 2002; Petitjean, 2003). Ce modèle nous permet d'exprimer l'activité économique générée localement et à court terme par la construction de la LGV SEA Tours-Bordeaux de trois façons différentes : production (output), valeur ajoutée et emplois. II s'appuie sur les principes des modèles de la théorie de la base économique puisqu'il cherche à mesurer la base exportatrice liée au chantier des régions traversées par la LGV SEA (effets directs et indirects) et son effet multiplicateur sur la sphère non basique de ces régions (effets induits).

\section{Méthode d'évaluation de l'impact économique}

\subsection{Expression générale du modèle économique}

Le modèle que nous retenons pour mesurer l'impact économique de la construction de la LGV SEA ToursBordeaux dans les régions traversées identifie le cheminement dans l'économie locale des dépenses de construction (Figure 1). Dans un premier temps, nous avons donc cherché à différencier les dépenses de construction s'étant diffusées localement (injections nettes) des dépenses de construction ayant été dépensées en dehors des régions traversées (fuites) (Lynch, 2000 ; Vollet et al., 2007 ; Barget et al., 2010). Dans un second temps, une fois les injections nettes identifiées, nous avons quantifié leur diffusion dans l'économie locale. Les injections nettes doivent être mesurées le plus finement possible car elles conditionnent en partie la mesure finale de l'impact économique. Comme le souligne Barget et Gouguet (2011), « au-delà de [...] différences relativement minimes, l'essentiel se joue au niveau du calcul des injections nettes, ce qui implique la collecte d'une information fiable sur le terrain ».

Au préalable, il est nécessaire de définir le champ des flux à prendre en compte dans le calcul de l'injection nette. Les différentes sources de financement du projet SEA font apparaître des financeurs extérieurs aux régions traversées (Etat, fonds privés) et des financeurs situés dans ces régions (collectivités locales). La question se pose alors de savoir si l'investissement des financeurs locaux dans le projet SEA représente une injection nette de revenu pour les régions traversées ou non. Dans le cadre d'une relance keynésienne à l'échelle d'un état et d'une région, le financement d'un investissement par l'emprunt ou la planche à billets peut être considéré comme faisant partie de revenus basiques. Dans ce cas, l'investissement consenti par les collectivités territoriales doit être comptabilisé comme une injection nette. En revanche, il existe un débat dans la littérature quant à la classification de l'investissement en revenus basiques ou non basiques lorsqu'il est financé par les recettes de la 
fiscalité locale. Pour Sombart (1902), les taxes et impôts locaux collectés auprès de la sphère basique doivent être considérés comme des revenus basiques. En revanche, Talandier (2012) considère que tous les traitements provenant d'impôts locaux constituent la base publique et sont donc des revenus basiques, sans chercher à savoir si ces impôts ont été collectés auprès de la sphère basique ou non basique. De leur côté, Segessmann et Crevoisier (2013) distinguent trois cas de figure pour les cantons suisses : les administrations locales et cantonales relèvent de l'économie résidentielle et de la sphère non basique tandis que l'administration fédérale est classée dans la sphère basique.

Ces différents positionnements montrent que la question de la classification des recettes et des dépenses des collectivités territoriales n'est pas tranchée. En outre, elle peut considérablement changer d'une collectivité territoriale à une autre en fonction de décisions politiques (stratégies de recours à l'emprunt, politiques fiscales, etc.). Dans ce contexte, la difficulté réside alors dans l'identification des recettes ayant financé les dépenses d'investissement. Les données pour l'année 2011 diffusées sur le site wwwlafinancepourtous.com ${ }^{4}$ indiquent qu'en moyenne $16 \%$ des recettes des collectivités territoriales françaises sont des recettes d'investissement (dont l'emprunt), que $24 \%$ sont issues des concours de l'Etat et que $50 \%$ proviennent des impôts locaux. Parallèlement, les collectivités territoriales françaises consacrent en moyenne $24 \%$ de leurs dépenses aux investissements. En considérant que l'intégralité des recettes d'investissements est consacrée aux dépenses d'investissements, et que les $8 \%$ restant sont financées par des concours de l'Etat, nous classons les dépenses d'investissement des collectivités locales dans la catégorie des revenus basiques. Ainsi, dans notre modèle, l'investissement dans la construction de la LGV SEA Tours-Bordeaux consenti par les collectivités territoriales des régions traversées représente une injection nette de revenus.

Figure 1 : Circuit économique de la construction de la LGV SEA Tours-Bordeaux

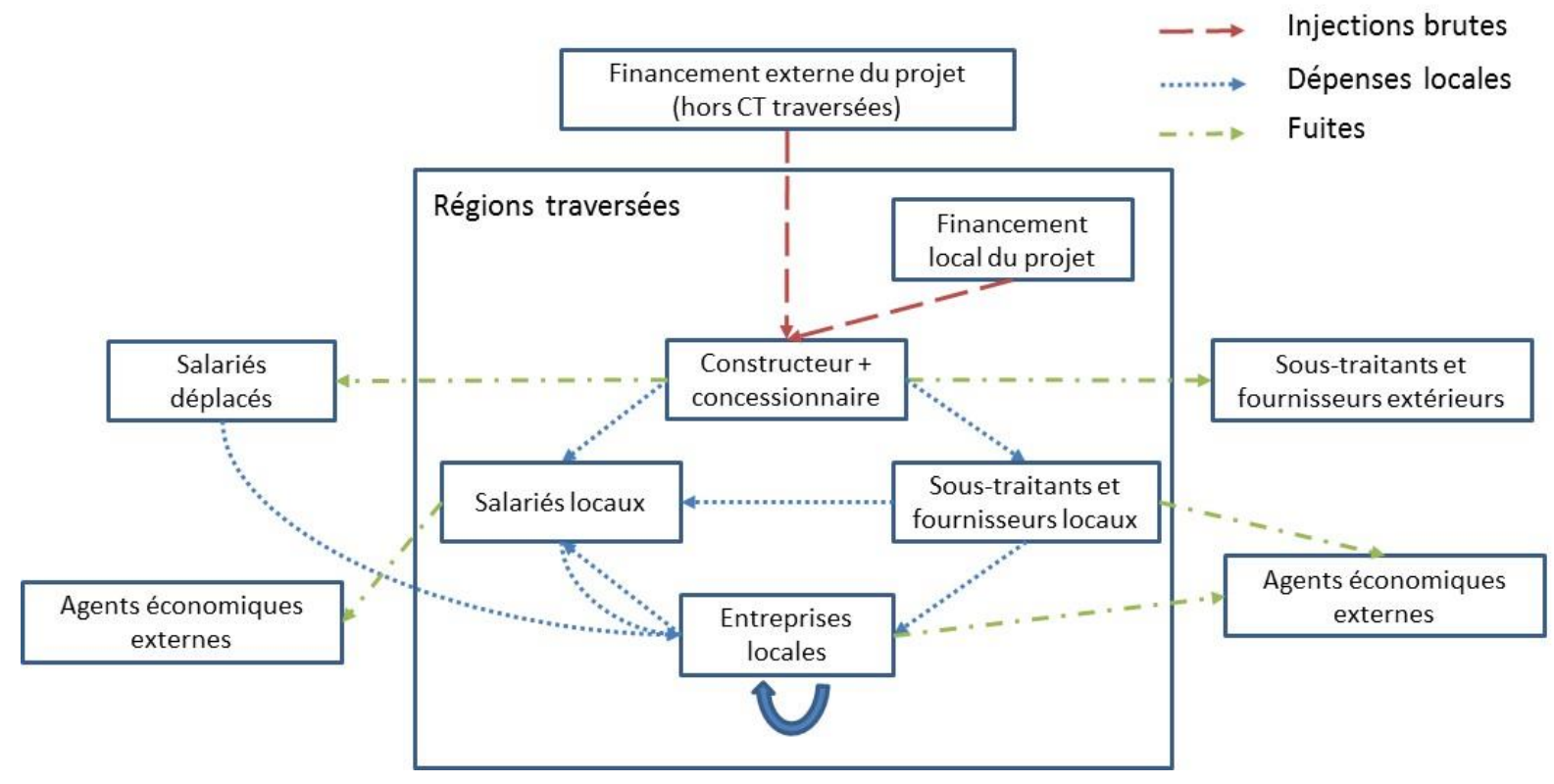

Nous calculons les injections nettes en sommant la masse salariale nette du constructeur et de ses principaux sous-traitants, et les consommations intermédiaires locales de celui-ci. A partir du montant des injections nettes et du coût total de la construction (injection brute), nous pouvons en déduire le montant des fuites. Au 15 mars 2013, l'avancement du projet SEA était de 41,5\%, soit environ 2,7 milliards d'euros courants d'injections brutes. $31 \%$ de ce montant a été dépensé localement, soit une injection nette d'environ 827 millions d'euros. Selon la convention de financement ${ }^{5}$ établie avant le début de la construction entre les différentes parties prenantes, la

\footnotetext{
$4 \mathrm{http} / / /$ www.lafinancepourtous.com/Decryptages/Dossiers/Impots-et-taxes-locales/La-fiscalite-locale-une-variable-cle-dansle-budget-des-collectivites

5 Convention de financement consultable à l'adresse Internet suivante : http://www.lacub.fr/sites/default/files/synchro_docs/ConseildeCommunaute/2011/02/11/P0D4K.pdf
} 
contribution des collectivités territoriales à cette injection nette est de l'ordre de $17 \%$. Celle des autres acteurs (Etat, acteurs privés) est estimée à $83 \%$.

Tableau 1 : Injections brutes et injections nettes dans les trois régions traversées (en euros courants)

\begin{tabular}{|c|c|c|c|}
\cline { 2 - 4 } \multicolumn{1}{c|}{} & $\begin{array}{c}\text { Collectivités } \\
\text { territoriales des } \\
\text { régions traversées }\end{array}$ & $\begin{array}{c}\text { Acteurs publics et } \\
\text { privés extérieurs aux } \\
\text { régions traversées }\end{array}$ & Total \\
\hline Injections brutes & $474578484 €$ & $2212546516 €$ & $2687125000 €$ \\
\hline Injections nettes & $148896007 €$ & $678304030 €$ & $827200036 €$ \\
\hline $\begin{array}{c}\text { Part des injections } \\
\text { nettes }\end{array}$ & $31 \%$ & $31 \%$ & $31 \%$ \\
\hline
\end{tabular}

L'injection nette IN dans la construction de la LGV SEA s'exprime de la manière suivante :

$$
I N=(M S+C)
$$

Où MS représente la masse salariale du constructeur et de ses principaux sous-traitants, $C$ représente le montant total des consommations intermédiaires locales du constructeur.

Suite aux injections nettes dans l'économie régionale, nous distinguons alors les effets direct $\left(B_{1}\right)$, indirect $\left(B_{2}\right)$ et induits $(R)$ et estimons l'impact économique total $(Y$ ) par la somme de ces trois composantes (Bouba-Olga, 2006). Nous mesurons ces grandeurs économiques en termes de production, de valeur ajoutée et d'emplois.

$$
Y=B_{1}+B_{2}+R
$$

Du point de vue de la théorie de la base économique, les effets directs $\left(B_{1}\right)$ et indirects $\left(B_{2}\right)$ constituent la base exportatrice tandis que les effets induits $(R)$ rendent compte de l'activité économique que génère cette base exportatrice lorsqu'elle est dépensée localement. Les effets directs, indirects et induits sont définis de deux manières différentes par les chercheurs. Certains considèrent que les effets directs sont observables, au moment où les dépenses dans le projet d'infrastructure de transport ont lieu, dans le secteur de la construction et dans les secteurs fournissant ce secteur en biens et services (Johnson et Thomas, 1990; OCDE, 2002). D'autres estiment que les effets directs sont « liés à la construction de la ligne, vont de la conception du projet à la plantation des espaces verts en passant par le dégagement des terrains, les terrassements, le montage des ouvrages d'art, la pose des équipements ferroviaires et la construction des bâtiments techniques. Ces emplois intègrent également les activités de suivi et de surveillance des travaux ainsi que les personnels des entreprises ne travaillant pas directement sur le site, comme les ingénieurs des bureaux d'études » (Berion et Sauter, 2011; Réseau Ferré de France, 2010). Dans cette seconde définition des effets directs, contrairement à la première, l'impact sur les fournisseurs de biens et services n'est pas pris en compte. II l'est en revanche dans les effets indirects qui « concernent la fabrication des fournitures nécessaires à la construction : matériaux d'extraction, ciment, énergie, transport, services, équipements ferroviaires (par exemple les longs rails soudés, les produits en béton préfabriqué...), etc.» (Berion et Sauter, 2011; Réseau Ferré de France, 2010). Pour Johnson et Thomas (1990) et l'OCDE (2002), les effets indirects correspondent à l'impact du projet sur les fournisseurs de biens et services des activités directs. Autrement dit, les effets indirects concernent les fournisseurs de rang 2 à $\mathrm{N}$. Les effets induits ont en revanche une définition commune. Ils correspondent à l'activité économique générée « lorsque les employés du secteur de la construction et d'autres secteurs de production [directement reliées aux dépenses du projet] dépensent leur revenu » (OCDE, 2002). Dans notre modèle, nous retenons la définition des effets directs et indirects de Bérion et Sauter, et de Réseau Ferré de France car elle différencie l'activité générée par le constructeur et ses sous-traitants intervenant sur le tracé de celle générée chez les fournisseurs, quel que soit leur rang dans la chaîne de production. On peut alors distinguer dans les régions traversées, l'impact du constructeur et des principaux sous-traitants (effet direct), l'impact des fournisseurs locaux (effets indirects) et 
l'impact des revenus versés aux salariés du constructeur et des fournisseurs locaux (effets induits). Finalement, le choix d'une définition plutôt qu'une autre n'a pas d'incidence sur l'estimation de l'impact total ni du multiplicateur de production. En revanche, le multiplicateur d'emploi variera selon la définition retenue. Les comparaisons que nous faisons avec d'autres travaux doivent donc être interprétées avec précaution.

Nous estimons ensuite l'effet multiplicateur $M$ de la construction de la LGV SEA sur les régions traversées en rapportant la production totale générée localement $Y$ sur l'injection nette IN. Cet indicateur permet de répondre à la question suivante : quelle production a été générée dans les régions traversées par la LGV SEA suite à l'injection nette de 1 euro?

$$
M=\frac{Y}{I N}
$$

Outre cet effet multiplicateur des injections nettes dans la construction sur la production locale, un multiplicateur d'emplois $M_{E}$ peut être calculé. II rapporte les emplois directs sur le total des emplois générés par la construction de la ligne (Catin et Nicolini, 2005). Plus largement, il est possible de mettre en perspective le nombre d'emplois indirects et induits générés pour un emploi direct. Le calcul de ces différents multiplicateurs permet notamment d'établir des comparaisons avec d'autres évaluations d'impact portant sur des objets similaires (grands chantiers d'infrastructure) ou différents, quand bien même les méthodologies retenues soient suffisamment proches pour être comparées.

$$
M_{E}=\frac{Y_{E}}{B_{1 E}}
$$

\subsection{Calcul des effets directs}

Pour obtenir la base exportatrice directe du chantier, autrement nommée " effets directs ", nous avons fait le choix de ne retenir que la masse salariale $M S$ du constructeur et de ses principaux sous-traitants entre le début du chantier et mars 2013 plutôt que la valeur ajoutée. En effet, comme les entreprises directement impliquées dans la construction ne sont pas situées dans les régions traversées, l'excédent brut d'exploitation n'est pas distribué localement. Ce choix méthodologique implique que la base exportatrice directe s'exprime de la même façon du point de vue de la production et de la valeur ajoutée. Mathématiquement, la base exportatrice directe $B_{1}$ s'écrit sous la forme matricielle de la manière suivante :

$$
B_{1}=M S
$$

Où MS représente le vecteur colonne répartissant la masse salariale du constructeur et de ses principaux soustraitants en $i$ branches d'activité.

L'estimation du nombre d'emplois directs engendrés par les injections nettes s'obtient à partir des données transmises par le constructeur. Ainsi, en sommant les effectifs mensuels du constructeur et de ses sous-traitants sur la période d'étude, puis en divisant le total par 12, nous obtenons le nombre d'emplois $B_{E 1}$ générés par la construction de la LGV SEA, en équivalents temps plein annuels ${ }^{6}$ (ETPA).

$$
B_{E 1}=\frac{\sum_{j=1}^{m} E_{j}}{12}
$$

Où $E_{j}$ représente le nombre d'emplois directs chez le constructeur et ses sous-traitants pour le mois $j$.

Parmi le total des emplois $B_{E 1}$, on distingue les emplois occupés par des personnels « locaux » et les emplois occupés par des personnels "déplacés ". Deux arguments justifient le choix de comptabiliser les emplois « déplacés » dans l'impact économique de la construction de la LGV SEA dans les régions traversées. Tout

\footnotetext{
${ }^{6}$ Derrière les termes d'emploi en équivalent temps plein annuel, nous entendons le nombre d'emplois correspondant à un temps plein d'une année que le chantier a généré. II ne faut pas confondre cette unité de mesure avec le nombre d'emplois (en ETP) occupés en moyenne pour chaque année du chantier.
} 
d'abord, « en se plaçant dans une perspective locale, un travailleur, même délocalisé, représente un emploi supplémentaire tout au long de la période de construction » (OCDE, 2002). Surtout, un emploi " déplacé » va générer des retombées économiques locales par le biais de dépenses de consommation (Fouqueray, 2014; Fouqueray et Lempereur, 2014).

\subsection{Calcul des effets indirects}

La base exportatrice indirecte (ou effets indirects) s'obtient quant à elle en sommant les productions locales générées aux différentes vagues de sous-traitance et de fourniture suite aux consommations intermédiaires locales initiales du constructeur. D'un point de vue matriciel, elle s'exprime de la manière suivante :

$$
B_{2}=(I-A)^{-1} \times C
$$

Où $C$ représente le vecteur colonne répartissant les consommations intermédiaires locales (hors sous-traitant classés dans la sphère directe) du constructeur en $i$ branches d'activités, $I$ représente une matrice identité de $i$ lignes et $i$ colonnes, $A$ représente la matrice carrée des coefficients techniques régionaux de dimension $i \times i$.

Afin d'obtenir la base exportatrice indirecte, en termes de valeur ajoutée, nous intégrons à l'équation précédente le vecteur colonne VA des taux de valeurs ajoutées nationaux en $i$ branches d'activités. L'utilisation de données nationales concernant le taux de valeur ajoutée par branche d'activité est susceptible de masquer les différences régionales quant à l'utilisation des facteurs de production et à leur prix. Face à l'absence de données sur les taux de valeurs ajoutées régionaux, nous avons fait le choix de ne pas tenir compte des écarts entre régions à ce sujet.

$$
B_{2}=(I-A)^{-1} \times C \times V A
$$

La base exportatrice indirecte $B_{2}$ étant égale à la somme des valeurs ajoutées des sous-traitants et fournisseurs de rang 1 à $\mathrm{N}$ du constructeur de chacune des $i$ branches d'activités, nous pouvons en déduire le nombre d'emplois en équivalent temps plein annuel (ETPA) générés au total et par branche d'activités en divisant $B_{2}$ par la productivité apparente du travail. Du point de vue de l'emploi, la base économique indirecte $B_{E 2}$ s'écrit alors :

$$
B_{E 2}=\sum_{i=1}^{n} \frac{B_{2 i}}{\text { prod }_{i t}}
$$

Où $B_{E 2}$ représente la base exportatrice (en valeur ajoutée) indirecte pour chacune des branches $i$, prodit représente la productivité apparente du travail pour chacune des branches $i$ pour l'ensemble $t$ des régions traversées.

\subsection{Calcul des effets induits}

L'effet des travaux de la LGV SEA sur l'économie locale ne se limite pas seulement aux effets directs et indirects. Les revenus dont disposent les personnes vont être à leur tour, pour partie, dépensés localement. Ces dépenses vont générer des effets induits que nous estimons également en termes de production, de valeur ajoutée puis d'emplois (en équivalent temps plein annuel). Notons $R_{1}$ les effets induits par la dépense locale des salariés directs et $R_{2}$ les effets induits par la dépense locale des salariés indirects. Nous faisons le choix de distinguer les calculs de $R_{1}$ et $R_{2}$ car la propension à consommer localement de ces deux types de salariés est sensiblement différente. Les salariés directs dépensent $42 \%$ de leur salaire net localement contre $54 \%$ pour les salariés indirects.

$$
R=R_{1}+R_{2}
$$

Avec 


$$
R_{1}=B_{1} \times r_{1} \times D \times(I-A)^{-1}
$$

Et

$$
R_{2}=B_{2} \times r_{2} \times D \times(I-A)^{-1}
$$

Où

$B_{1}$ représente la base exportatrice directe, $r_{1}$ représente la part moyenne du salaire des salariés directs du chantier consacré à des consommations locales, $D$ représente le vecteur colonne répartissant la consommation effective des ménages en $i$ branches d'activités, $I$ représente une matrice identité de $i$ lignes et $i$ colonnes, $A$ représente la matrice carrée des coefficients techniques régionaux de dimension $i \times i, B_{2}$ représente la base exportatrice indirecte, $r_{2}$ représente la part moyenne du salaire des salariés indirects du chantier consacré à des consommations locales.

Afin d'obtenir les effets induits en termes de valeur ajoutée nous intégrons aux deux équations précédentes le vecteur colonne VA des taux de valeurs ajoutées nationaux en $i$ branches d'activité. Les effets induits $R_{1}$ et $R_{2}$ étant égaux à la somme des valeurs ajoutées des entreprises fournissant des biens et services aux personnels directs et indirects impactés par l'activité du chantier SEA, nous pouvons en déduire le nombre d'emplois (en équivalent temps plein annuel) générés en divisant $R_{1}$ et $R_{2}$ par la productivité apparente du travail. Du point de vue de l'emploi, les effets induits s'écrivent alors :

$$
R_{E}=\sum_{i=1}^{n} \frac{R_{1 i}+R_{2 i}}{\operatorname{prod}_{i t}}
$$

Où $R_{1 i}$ représente les effets induits (en valeur ajoutée) générés par la base exportatrice directe pour chacune des branches $i, R_{2 i}$ représente les effets induits (en valeur ajoutée) générés par la base exportatrice indirecte pour chacune des branches $i$, prodit représente la productivité apparente du travail pour chacune des branches $i$ pour l'ensemble $t$ des régions traversées.

\subsection{Les différentes hypothèses du modèle}

Les résultats du modèle sont fortement dépendants de la propension des entreprises locales à dépenser localement les revenus qu'elles ont captés grâce à la construction de la LGV SEA Tours-Bordeaux. Dans l'expression du modèle, cette propension à dépenser localement des entreprises locales est intégrée dans la matrice $A$ des coefficients techniques régionaux. En effet, cette matrice $A$ des coefficients techniques régionaux s'obtient par multiplication des coefficients de la matrice $T$ des coefficients techniques nationaux avec ceux du vecteur colonne $G$ des propensions des entreprises locales à dépenser régionalement (Catin et Nicolini, 2005). Autrement écrit, si

$$
T=\left[\begin{array}{ccc}
t_{11} & \cdots & t_{1 j} \\
\vdots & \ddots & \vdots \\
t_{i 1} & \cdots & t_{i j}
\end{array}\right]
$$

et

$$
G=\left[\begin{array}{c}
g_{11} \\
\vdots \\
g_{i 1}
\end{array}\right]
$$

Alors la matrice $A$ des coefficients techniques régionaux s'exprime de la façon suivante :

$$
A=\left[\begin{array}{ccc}
t_{11} \times g_{11} & \cdots & t_{1 j} \times g_{11} \\
\vdots & \ddots & \vdots \\
t_{i 1} \times g_{i 1} & \cdots & t_{i j} \times g_{i 1}
\end{array}\right]=\left[\begin{array}{ccc}
a_{11} & \cdots & a_{1 j} \\
\vdots & \ddots & \vdots \\
a_{i 1} & \cdots & a_{i j}
\end{array}\right]
$$


Afin de rendre compte de l'influence de la propension des entreprises locales à se fournir et à sous-traiter localement dans l'évaluation de l'impact économique, nous retenons quatre hypothèses sur la valeur des coefficients du vecteur colonne $G$. En conséquence, la matrice carrée $A$ des coefficients techniques régionaux prendra à son tour quatre formes différentes que nous différencierons en les nommant $A_{1}, A_{2}, A_{3}$ et $A_{4}$. Etant donné que cette matrice entre dans le calcul des effets indirects et des effets induits, nous obtenons donc, au total, 16 estimations d'impact économique différentes.

Tout d'abord, nous retenons deux valeurs extrêmes de la propension des entreprises régionales à se fournir et à sous-traiter localement: $100 \%$ et $0 \%$. Dans le premier cas, la matrice carrée $A_{2}$ des coefficients techniques régionaux sera égale à la matrice carrée $T$ des coefficients techniques nationaux. Dans le second cas, la matrice carrée $A_{1}$ des coefficients techniques régionaux sera une matrice nulle.

Nous faisons également une troisième hypothèse, que nous qualifions d'intermédiaire basse, selon laquelle la propension des entreprises locales à se fournir localement est égale à celle du constructeur, et ce pour chacune des $i$ branches d'activité étudiées. Selon cette hypothèse, la propension du constructeur à dépenser localement renseigne sur la capacité du territoire à répondre à une demande locale. Bien qu'elle comporte des biais liés à l'influence des choix commerciaux du constructeur et au caractère spécifique du secteur étudié (les travaux publics), cette hypothèse a le mérite de s'appuyer sur des données précises pour un volume d'activité non négligeable. Au total, au 15 mars 2013, le constructeur a dépensé plus de 1,3 milliard d'euros en sous-traitance et en fourniture (hors sous-traitant classés dans la sphère directe) dont $42 \%$ dans les trois régions traversées.

Enfin, la quatrième et dernière hypothèse s'appuie sur la méthode du besoin minimal (Ullman et Dacey, 1960) qui est un approfondissement du coefficient de localisation (Catin et Nicolini, 2005; Richardson, 1985; Vollet et Dion, 2001). Cette méthode consiste à comparer la structure économique d'un territoire, en termes d'emplois, avec celles d'autres territoires de tailles comparables afin d'en déduire le rapport entre les activités basiques et les activités non basiques sur le territoire d'étude et ainsi estimer la propension à dépenser localement des agents économiques locaux. Le recours à cette méthode nécessite de faire les hypothèses que " la productivité de la région étudiée et celle de l'échelon géographique de référence (la région minimale) soient égales et que les modes de consommation de la région et ceux de la région minimum soient proches " (Vollet et Dion, 2001). Nous qualifions de région minimum celle où la part des emplois de la branche d'activité $i$ dans l'emploi total est la plus faible. Nous considérons alors que l'intégralité de la production de cette branche permet d'alimenter les besoins de cette même région. A l'inverse, dans les autres régions comparables, la branche d'activité $i$ exporte une partie de sa production, générant des emplois basiques. Le nombre d'emplois basiques $X_{i r}$ pour la branche d'activité $i$ est alors égal au produit de l'emploi total $E_{r}$ de la région $r$ et de la différence entre le taux d'emplois dans la branche $i$ dans la région étudiée et le taux d'emplois dans la branche $i$ dans la région minimale.

$$
X_{i r}=\left(\frac{E_{\text {ir }}}{E_{r}}-\frac{E_{\text {im }}}{E_{m}}\right) E_{r}
$$

$E_{\text {ir }}$ représente le total des emplois de la branche $i$ de la région étudiée $r, E_{r}$ représente le total des emplois de la région étudiée $r, E_{i m}$ représente le total des emplois de la branche $i$ de la région minimum $m, E_{m}$ représente le total des emplois de la région minimum $m$.

Finalement le total des emplois basiques $X_{r}$ de la région étudiée $r$ correspond à la somme des emplois basiques $X_{\text {ir }}$ de chaque branche d'activité $i$.

$$
X_{r}=\sum_{i=1}^{n} X_{i r}
$$

Chaque coefficient du vecteur colonne $G$ des propensions des entreprises locales à dépenser localement s'obtient en faisant le rapport, pour la région étudiée, entre le nombre d'emplois basiques dans la branche considérée et le nombre d'emploi total dans cette même branche en région.

$$
g_{i}=\frac{X_{i r}}{E_{i r}}
$$


Dans notre recherche, nous avons rassemblé les 22 régions françaises métropolitaines, hors île-de-France, en 7 grandes régions (Figure 2) de sorte que leur taille, en termes d'emplois, soit comparable. Parmi ces 7 grandes régions, il y a bien évidemment celle regroupant les 3 régions traversées par la LGV SEA Tours-Bordeaux. La propension à dépenser localement moyenne que nous obtenons est de $82 \%$ et oscille entre $42 \%$ et $100 \%$ d'une branche d'activité à l'autre. Un test de sensibilité réalisé avec un autre regroupement de régions confirme ce résultat avec une propension à dépenser localement de $83 \%$. Malgré cela, la méthode du besoin minimal a tendance à surestimer la part des productions d'une région consommée localement puisqu'elle suppose que toutes les régions exportent mais aucune n'importe (Pratt, 1968; Richardson, 1985). Aussi, les coefficients techniques régionaux obtenus grâce à cette méthode peuvent être considérés comme relevant d'une hypothèse intermédiaire haute du modèle.

Figure 2 : Cartographie des régions retenues pour la méthode du besoin minimal

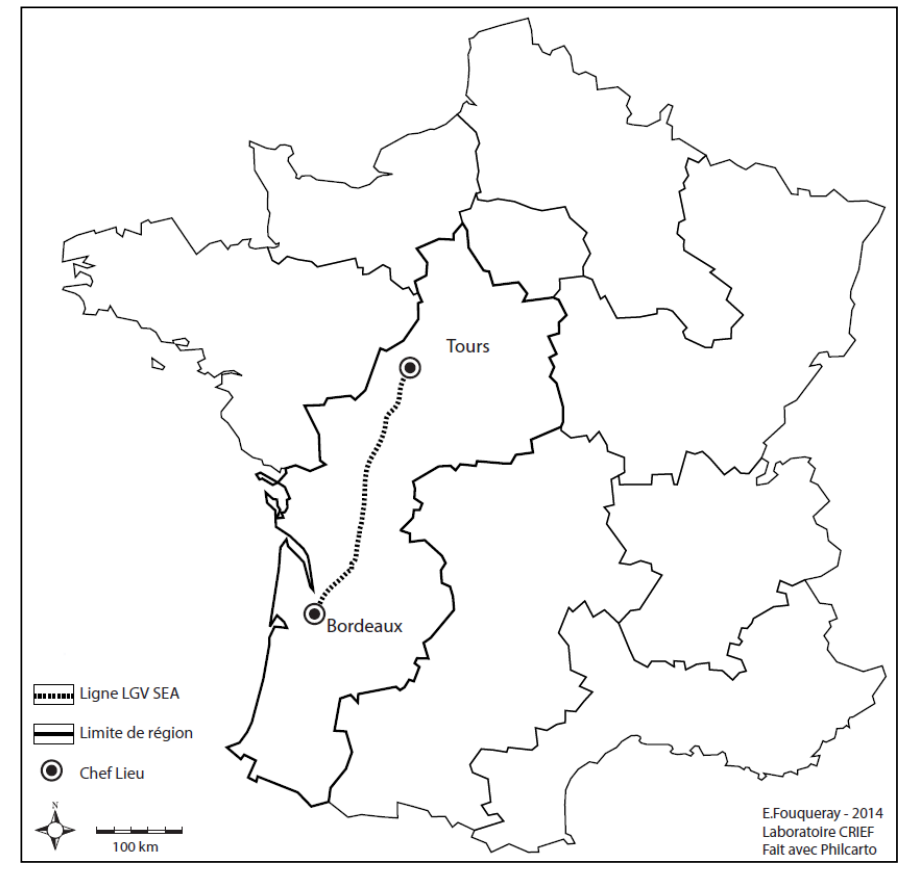

\section{Les données mobilisées pour l'estimation des paramètres}

La modélisation de l'impact économique et de ses différents effets a nécessité de mobiliser des données existantes, notamment diffusées par l'INSEE, mais également des données issues d'enquêtes conçues spécifiquement à cet effet. II s'agit donc d'un modèle hybride (Richardson, 1985) dont l'objectif est d'associer la précision des données nationales sur les relations entre branches d'activités tout en tenant compte des spécificités locales influençant fortement l'intensité de l'impact économique. Pour chacun des effets que nous estimons, nous détaillons dans cette partie les données mobilisées.

\subsection{Les données mobilisées pour le calcul des effets directs}

Les effets directs B1 rendent compte de l'impact local du constructeur et des sous-traitants intervenant directement sur le chantier. Ils s'évaluent par la masse salariale et le nombre d'emplois de ces agents économiques. Pour faire ces estimations, nous avons mobilisé, dans un premier temps, des données précises collectées directement auprès du constructeur concernant l'évolution du coût de la construction et des effectifs du projet. Dans un second temps, nous avons mené une enquête auprès de 909 salariés directs du projet SEA afin 
d'estimer leur salaire net mensuel. En combinant ces deux sources de données, nous avons calculé la masse salariale directe du projet ainsi que les emplois générés.

\subsection{Les données mobilisées pour le calcul des effets indirects}

La première source de données utilisée pour estimer les effets indirects provient du constructeur de la LGV SEA. II s'agit d'une base de données sur ses consommations intermédiaires qui recense l'ensemble des contrats de sous-traitance et de fourniture passés depuis le début du chantier jusqu'à mars 2013. Nous avons enrichi cette base avec des données sectorielles et géographiques. Ainsi, pour chaque contrat passé, nous connaissons l'entreprise co-contractante, sa localisation et son secteur d'activité. A partir de ces informations, nous avons constitué le vecteur colonne $C$ répartissant les consommations intermédiaires (hors sous-traitant classés dans la sphère directe) du constructeur sur le territoire d'étude en 17 branches d'activité.

Nous avons ensuite estimé les effets d'entrainement locaux, dans les différentes branches d'activité, des dépenses locales du constructeur en mobilisant une matrice de Leontief. Pour cela, nous avons utilisé des données de l'INSEE pour l'année 2009 sur les Tableaux Entrées Sorties (TES) de la comptabilité nationale en 17 branches d'activité. Nous avons d'abord retenu la matrice $A$ des coefficients techniques issus de ces tableaux que nous avons corrigés selon les quatre hypothèses précédemment détaillées. Puis, pour estimer les effets indirects en termes de valeur ajoutée, nous avons utilisé les taux de valeur ajoutée par branche d'activité renseignés dans ces Tableaux Entrées Sorties.

Enfin, pour calculer le nombre d'emplois indirects générés localement, nous avons calculé la productivité apparente du travail prod ${ }_{i t}$ pour chacune des 17 branches i pour l'ensemble $t$ des trois régions traversées. Nous nous sommes appuyés sur des données nationales de l'INSEE pour l'année 2009, auxquelles nous avons appliqué un coefficient correcteur régional à partir de données régionales de l'INSEE sur l'emploi et la valeur ajoutée. En moyenne, sur l'ensemble des branches d'activités, il apparaît que la productivité apparente du travail sur l'ensemble des trois régions traversées est de 8 points de pourcentage inférieure à celle du niveau national.

\subsection{Les données mobilisées pour le calcul des effets induits}

Le calcul des effets induits s'est effectué en deux temps. Nous avons d'abord évalué les effets induits $R_{1}$ générés par la dépense des revenus des salariés directs $\left(B_{1}\right)$ puis nous avons ensuite mesuré les effets induits $R_{2}$ engendrés par la dépense des revenus des salariés indirects $\left(B_{2}\right)$. En plus des montants de $B_{1}$ et $B_{2}$, l'expression de ces deux types d'effets induits diffère quant aux valeurs des coefficients $r_{1}$ et $r_{2}$.

Pour estimer la propension à dépenser localement $r_{1}$, nous avons procédé à une enquête auprès de 909 salariés du chantier LGV SEA. Nous les avons questionnés sur le niveau de leur salaire net mensuel, toute prime comprise, ainsi que sur la part de ce salaire dépensée localement. A partir des réponses obtenues, nous avons calculé le salaire net mensuel moyen et le montant moyen des consommations locales en tenant compte du poids des différentes catégories socio-professionnelles dans l'emploi direct et de son évolution dans le temps, ainsi que du profil des salariés (en déplacement ou locaux). Nous avons finalement obtenu $r_{1}$ en rapportant le montant moyen des consommations locales sur le salaire net mensuel moyen.

La détermination de la propension à dépenser localement $r_{2}$ à quant à elle été obtenue en combinant une partie des résultats de l'enquête auprès des 909 salariés du chantier et des données de l'INSEE issues du recensement de la population en 2009 et traitant des «Flux de mobilité - déplacements domicile-travail ». Plus précisément, à partir de l'enquête auprès des salariés du chantier, nous avons calculé la part moyenne des consommations locales dans le salaire net selon les profils de salariés du chantier (locaux ou en déplacement). Nous avons considéré que cette part était constante pour un même profil quel que soit le secteur d'activité et pouvait donc être généralisée à l'ensemble des salariés du territoire étudié. En parallèle, grâce aux données de I'INSEE, nous avons recensé le nombre d'actifs qui travaillent dans les régions traversées par la LGV SEA ainsi que leur territoire de résidence. Nous avons alors estimé la part des salariés locaux et la part des salariés déplacés dans l'emploi total du territoire d'étude. Finalement, il apparaît que la différence entre l'estimation de $r_{1}$ 
et de $r_{2}$ réside exclusivement dans le fait que la part des salariés locaux de la sphère indirecte est plus forte (98\%) que celle de la sphère directe $(53 \%)$.

Enfin, les paramètres $A, B$ et $V A$ étant commun à $R_{1}$ et $R_{2}$, nous les avons estimé à partir des mêmes sources de données et selon la même méthode. Nous avons mobilisé une nouvelle fois les Tableaux Entrées et Sorties de la comptabilité nationale pour l'année 2009 en 17 branches d'activité produit par l'INSEE. A partir de ces derniers et de la même manière que pour la mesure des effets indirects, nous avons ensuite fait varier la matrice des coefficients techniques régionaux $A$ selon les quatre hypothèses décrites précédemment. En revanche, les vecteurs colonnes $B$ et $V A$, représentant respectivement la consommation effective des ménages et le taux de valeur ajoutée par branche d'activité, tiennent compte exclusivement de données nationales.

\section{Principaux résultats du modèle}

\subsection{Le chantier de la LGV SEA Tours-Bordeaux : éléments de contexte}

Au 15 mars 2013, 1,3 milliard d'euros de contrats de sous-traitance et de fourniture avaient été passés par le constructeur, dont $43 \%$ avec des entreprises situées dans les trois régions traversées. Au total, ce sont plus de 350 entreprises locales qui ont donc contribué à la construction de la LGV SEA sur la période étudiée. En parallèle, les effectifs du chantier ont continuellement progressé pour atteindre près de 7500 personnes en mars 2013 (Figure 3).

Figure 3 : Evolution des effectifs de la construction de la LGV SEA Tours-Bordeaux de juillet 2011 à mars 2013

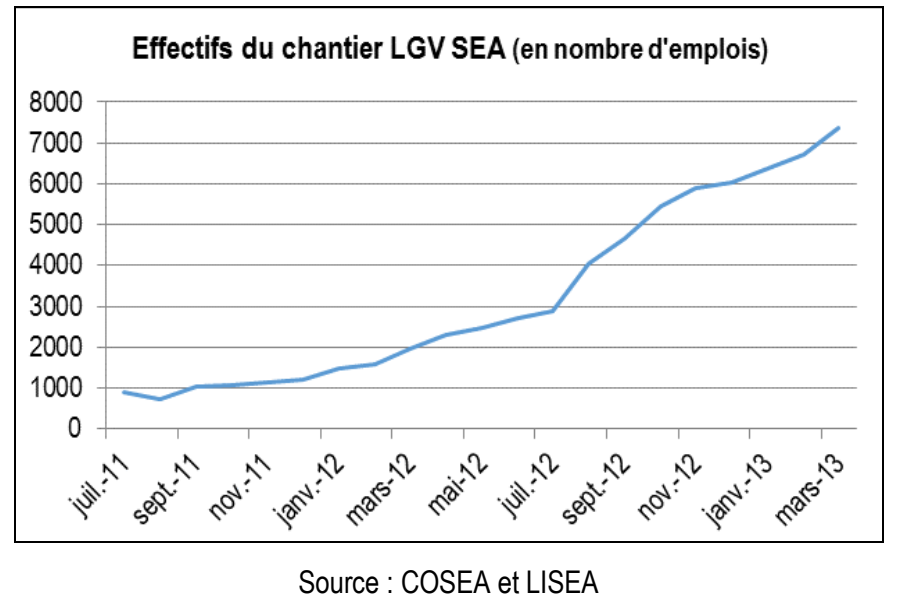

Selon l'enquête que nous avons conduite auprès de 909 personnels du projet, dont les résultats ont été confirmés par les données du constructeur, les effectifs se composent pour une moitié de salariés locaux $(53 \%)$ et pour une autre moitié de salariés déplacés (47\%). Le panier moyen de consommation locale des personnels varie d'une catégorie socio-professionnelle à une autre (Fouqueray, 2014; Fouqueray et Lempereur, 2014). Les cadres dépensent en moyenne 1307 euros localement et mensuellement, tandis que les compagnons consomment en moyenne 930 euros. Ces dépenses locales génèrent de l'activité et de la richesse pour les agents économique locaux offrant des biens et services aux ménages (logement, alimentation, transport, loisir, habillement, etc.). Le modèle d'impact économique que nous avons décrit dans la partie précédente nous permet d'estimer l'effet total de la mobilisation du tissu économique local sur le développement des trois régions traversées par la LGV SEA. 
4.2. Confrontation des différents résultats du modèle: une relative stabilité pour les hypothèses intermédiaires

Nous avons fait le choix de modéliser l'impact économique de court terme de la construction de la LGV SEA selon plusieurs hypothèses quant à la valeur des coefficients techniques régionaux afin de tester l'influence de cette variable sur le résultat final mais également de repérer les ressemblances et dissemblances entre les méthodes d'estimation de ces coefficients. Le tableau suivant rend compte des résultats obtenus avec l'une ou l'autre des hypothèses et permet de répondre à nos interrogations. La relative stabilité de l'impact économique en fonction des deux estimations intermédiaires des coefficients techniques régionaux semble témoigner de la robustesse des résultats obtenus.

Tableau 2 : Impact économique de la construction de la LGV SEA selon les quatre hypothèses retenues

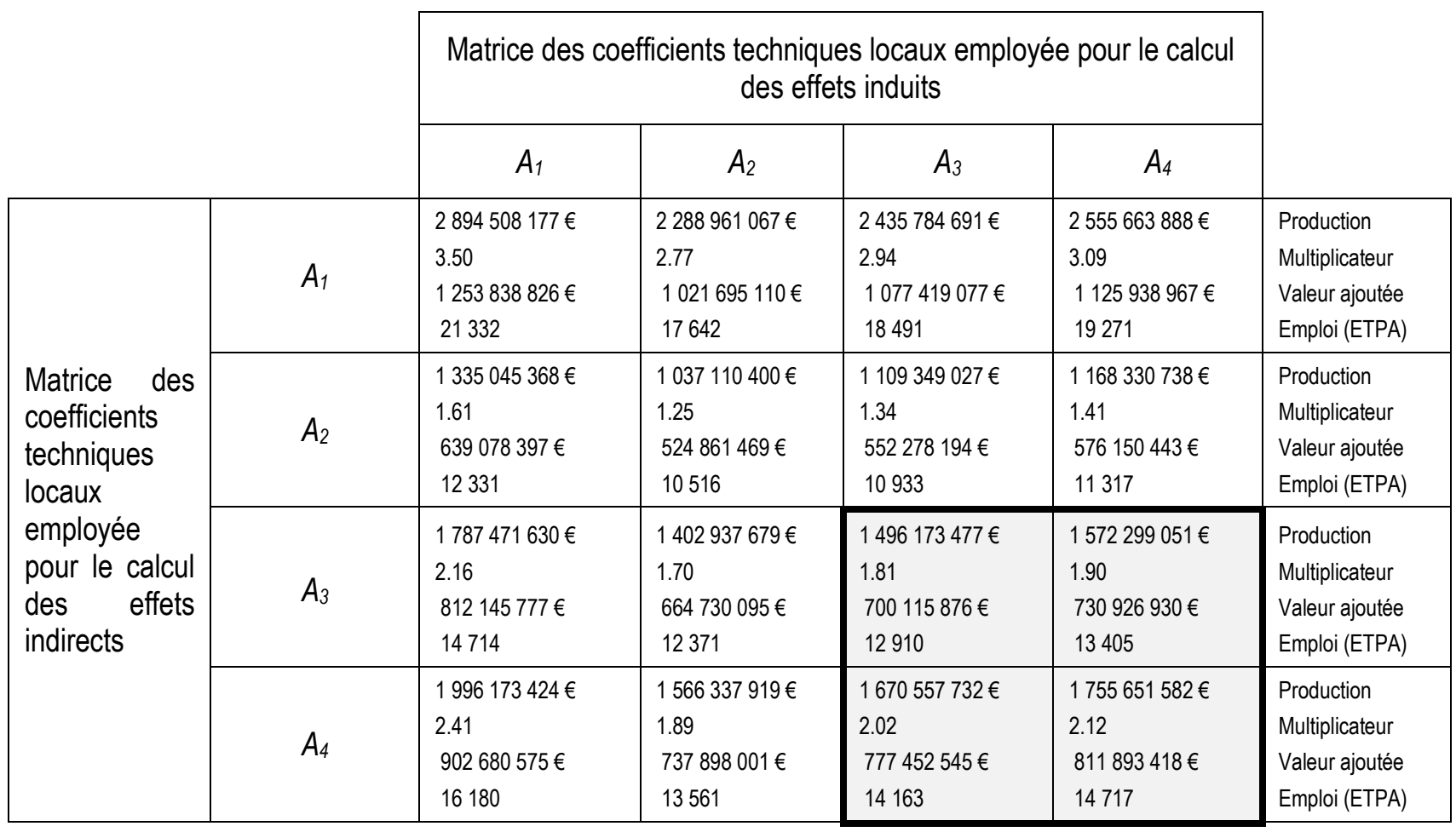

Lorsque l'on retient des valeurs extrêmes pour estimer les coefficients techniques régionaux $(0 \%$ et $100 \%)$, l'impact économique varie du simple au double: $1037110400 €$ de production générée et 10516 emplois totaux au minimum et $2894508177 €$ et 21332 emplois au maximum. Le multiplicateur varie quant à lui de 1,25 , au minimum, à 3,5 au maximum. Bien entendu, ces deux valeurs extrêmes ne représentent pas la réalité de l'impact économique pour les régions traversées. Celle-ci se situe entre ces deux bornes, à un niveau que nous avons tenté de déterminer en estimant les coefficients techniques régionaux à partir des données du constructeur et de la méthode du besoin minimal. Selon ces hypothèses (cases grises du tableau 2), la dépense initiale du constructeur dans les entreprises des régions traversées a généré, du point de vue de l'emploi, entre 12910 et 14717 ETPA sur l'ensemble de la période ${ }^{7}$. La production totale est, quant à elle, comprise entre 1,5 et 1,75 milliards d'euros tandis que la valeur ajoutée s'échelonne entre 700 et 811 millions d'euros. Enfin, l'effet multiplicateur fluctue entre 1,81 et 2,12. Ce résultat nous apporte un premier enseignement sur l'intérêt de comparer les différentes méthodes d'estimation des modèles d'impact, et en particulier des coefficients

\footnotetext{
7 Parmi ces emplois, on recense des emplois que la construction de la LGV SEA a contribué à maintenir ainsi que des emplois qui ont été créés. L'analyse que nous faisons ne nous permet pas de nous prononcer sur la part de chacune de ces deux catégories d'emplois générés.
} 
techniques régionaux, afin d'affiner au mieux l'évaluation. A ce titre, les résultats d'une enquête 8 conduite en 2014 auprès de sous-traitants et de fournisseurs du constructeur situés sur le territoire d'étude a mis en évidence que $67 \%$ de leurs consommations intermédiaires étaient orientées vers des entreprises locales, soit 15 points de pourcentage de moins que le coefficient obtenu à partir de la méthode du besoin minimal (82\%) et 25 points de pourcentage de plus que le coefficient obtenu à partir des données du constructeur (42\%). Considérant que les estimations les plus probables du modèle (cases grises du tableau 2) étaient suffisamment robustes et proches, nous avons fait le choix de présenter l'impact économique du chantier de la LGV SEA sous la forme d'une moyenne de ces quatre solutions afin de le comparer avec ceux obtenus dans d'autres travaux de recherche portant sur le même objet.

\subsection{Déclinaison de l'impact économique par type d'effets et comparaison avec d'autres travaux}

Entre le début du chantier en 2011 et mars 2013, la construction de la LGV SEA Tours-Bordeaux a permis une création de richesse dans les trois régions traversées d'environ 755 millions d'euros. Cette richesse, correspondant à la valeur ajoutée locale du chantier, s'est traduite par un volume de production de 1,6 milliards d'euros et 13799 emplois locaux (en ETPA). Elle est le fruit d'un effet multiplicateur à court-terme de 1,96 des injections nettes (827 millions d'euros) sur la production. Ce multiplicateur est proche de ceux diffusés dans un rapport sur l'impact économique et financier des dépenses d'infrastructure aux Etats-Unis (Cohen et al., 2012). S'appuyant sur des données du Bureau of Economic Analysis qui actualise régulièrement les Tableaux Entrées et Sorties de l'économie Américaine à l'échelle fédérale et régionale, il rend compte d'un effet multiplicateur variant entre 1,9 et 2,1 selon les années (entre 1998 et 2009). On peut supposer que d'une région à l'autre ce multiplicateur fluctue en fonction de la structure économique régionale. Cependant, le fait que ce résultat moyen pour l'économie nationale Américaine soit proche de celui obtenu dans le cadre de notre modèle tend à valider la méthode retenue et à confirmer le niveau de l'impact des grands chantiers sur les économies régionales. Une autre étude américaine portant sur l'impact économique local de la construction de l'Highway 17 fait état d'un multiplicateur de production de 3,7. Ce multiplicateur est voisin de celui que nous obtenons $(3,5)$ lorsque nous retenons comme hypothèse que les consommations intermédiaires des sous-traitants et fournisseurs locaux sont $100 \%$ locales (Tableau 2).

Tableau 3 : Effets directs, indirects et induits de la construction de la LGV SEA Tours-Bordeaux

\begin{tabular}{|l|c|c|c|}
\cline { 2 - 4 } \multicolumn{1}{c|}{} & Production & Valeur ajoutée & Emplois (ETPA) \\
\hline Effets directs (B1) & $\begin{array}{c}198375967 € \\
(0 €)\end{array}$ & $\begin{array}{c}198375967 € \\
(0 €)\end{array}$ & $\begin{array}{c}5663 \\
(0)\end{array}$ \\
\hline \multirow{2}{*}{ Effets indirects (B2) } & $999379447 €$ & $372154824 €$ & 5656 \\
& $(75911738 €)$ & $(33842855 €)$ & $(581)$ \\
\hline \multirow{2}{*}{ Effets induits (R) } & $425915046 €$ & $184566401 €$ & 2480 \\
& $(54047540 €)$ & $(22281487 €)$ & $(343)$ \\
\hline \multirow{2}{*}{ Impact total (Y) } & $\mathbf{1 6 2 3 6 7 0 4 6 1 €}$ & $\mathbf{7 5 5 0 9 7 1 9 2 €}$ & $\mathbf{1 3 7 9 9}$ \\
& $(\mathbf{1 1 3 3 0 1 8 6 1 € )}$ & $(\mathbf{4 9} 439255 €)$ & $(\mathbf{8 0 0})$ \\
\hline
\end{tabular}

Lecture : la production, la valeur ajoutée et l'emploi ont été obtenus en faisant la moyenne des 4 hypothèses les plus probables. Le nombre entre parenthèses correspond à l'écart-type.

Hormis pour l'emploi, les effets directs (production et valeur ajoutée) sont inférieurs aux effets indirects. Ce résultat s'explique par le fait que seule la masse salariale a été retenue dans le calcul de la valeur ajoutée et de la production du constructeur. Les autres grandeurs économiques contribuant à la production et à la valeur

\footnotetext{
8 Cette enquête qui a débuté en mars 2014 et s'est terminée en septembre 2014 cible environ 330 entreprises soustraitantes et fournisseuses du constructeur. Elle a recueilli, au moment où est rédigé cet article, 104 réponses. 34 entreprises ont accepté d'indiquer les montants et l'origine géographique de leurs consommations intermédiaires, aboutissant au ratio de $67 \%$ que nous mentionnons.
} 
ajoutée n'ont pas été prises en compte du fait de la non-localisation du constructeur dans les 3 régions traversées. Le constructeur a déplacé ses équipes et ses machines pour travailler sur le chantier, ce qui laisse augurer des retombées économiques pour les régions où sont situés les établissements du constructeur et le domicile principal des salariés. Les seules retombées locales, en termes de production et de valeur ajoutée, sont donc celles générées par l'emploi du constructeur pendant le chantier (Figure 4).

Figure 4 : Diagramme de Sankey représentant le processus de création de richesses liée à la construction de la LGV SEA dans les trois régions traversées

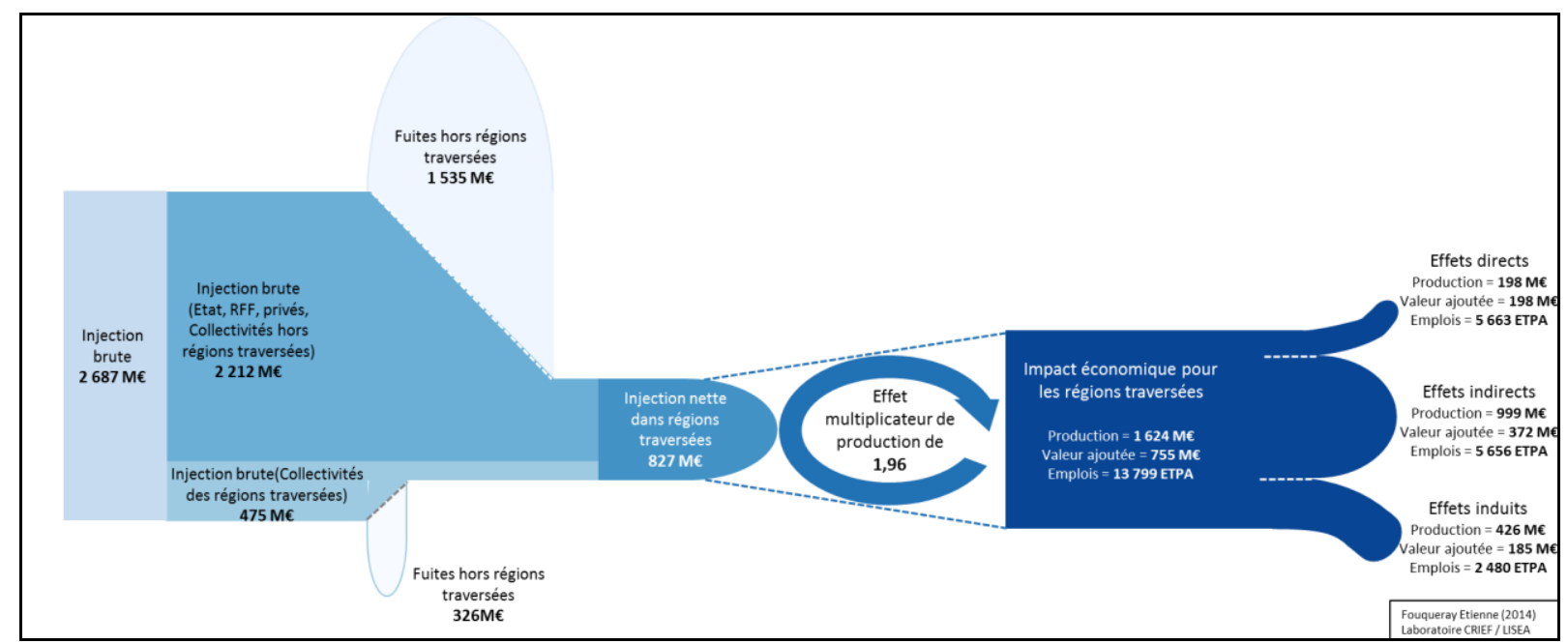

Pour un emploi direct occupé, la construction de la LGV SEA a généré 1 emploi indirect et 0,44 emploi induit localement. Nous obtenons donc un multiplicateur d'emplois de 2,44. Ce multiplicateur d'emplois est proche de celui que l'on retrouve dans plusieurs études d'impact portant sur des grands chantiers. Dans leur rapport sur l'impact de la construction de l'Highway 17 (2008) Wubneh et ses collègues calculent un multiplicateur d'emploi de 2,8 tandis qu'un rapport de l'OCDE (2002), souvent pris en référence pour estimer les emplois générés par des grands projets (Réseau Ferré de France, 2010), l'évalue à 2,7. Selon cette dernière étude, il existe un multiplicateur de 1,016 entre le nombre d'emplois directs et le nombre d'emplois indirects et un multiplicateur de 0,66 entre le nombre d'emplois directs et le nombre d'emplois induits. Les différences de multiplicateurs d'emplois peuvent alors s'expliquer par i) des écarts de productivité du travail entre territoires, entre branches d'activité, et entre années de construction ii) des écarts dans la propension des agents économiques locaux à consommer localement, iii) des écarts de coûts des facteurs de production, iv) des écarts au niveau des technologies de production, v) la taille du territoire d'étude, vi) l'horizon temporel de l'évaluation.

\subsection{L'impact économique de la construction de la LGV SEA par branche d'activité}

Du fait des relations entre branches d'activité, la construction de la LGV SEA a mobilisé toutes les branches d'activité de l'économie régionale avec plus ou moins d'intensité (Tableau 3). Sans surprise et confirmant des travaux passés (Cohen et al., 2012; Wubneh, 2008), le secteur le plus impacté en termes de richesse et d'emplois générés est celui de la construction. Arrivent ensuite les secteurs fournissant le secteur de la construction en machines et équipements (Fabrication d'autres produits industriels), en matières premières et produits issus d'une première transformation (Industries extractives, Fabrication d'autres produits industriels) et en matière grise (Activités scientifiques et techniques). La mobilisation de ces secteurs témoigne du poids des effets directs et indirects dans l'impact économique du chantier pour les régions traversées. Cela étant, les effets induits par les dépenses de consommation des revenus directs et indirects ne sont pas négligeables et sont à l'origine de la mobilisation de branches d'activité tournées vers la demande des ménages (Agriculture, Fabrication de denrées alimentaires, hébergement et restauration, Commerce). Au total, la construction de la 
LGV SEA a généré de l'activité économique et des emplois principalement dans des secteurs productifs privés de l'économie régionale.

Dans l'ensemble des trois régions traversées par la LGV SEA, les branches d'activités les plus impactées, en termes d'emplois, par la construction de la ligne ne sont pas celles qui sont les plus présentes dans le tissu économique local. Lorsque l'on met en perspective l'indice de spécialisation sectorielle ${ }^{9}$ des trois régions traversées et le poids de chaque branche d'activité dans l'impact économique du chantier, on observe de nombreuses différences. Alors que le secteur de la construction représente $47 \%$ des emplois générés par le chantier de la LGV SEA, il ne représente que 7\% des emplois du territoire d'étude (en référence à l'année 2011). On observe un résultat similaire pour le secteur de la fabrication d'autres produits industriels (métallurgie, éléments en béton, béton prêt à l'emploi, etc.). A l'inverse, les emplois publics (branche de l'administration publique, de l'enseignement, de la santé humaine et de l'action sociale) pèsent un tiers des emplois du territoire d'étude et seulement $2 \%$ des retombées économiques du chantier. Le secteur du commerce est lui aussi sousreprésenté dans les secteurs impactés par le chantier de la LGV SEA comparativement à son poids dans l'économie locale. Au-delà de la simple observation de la répartition inégale des impacts économiques locaux de la construction de la LGV SEA, on peut se questionner sur son effet structurant de long terme pour les branches les plus impactées. Plusieurs auteurs ont mis en évidence l' " effet bouffée d'oxygène » des grands chantiers d'infrastructures pour les entreprises locales et le risque de "surchauffe » puis de "chute brutale de l'activité à la fin du chantier » (Berion, 2002; Burmeister, 1997; Petitjean, 2003). Ces travaux tendent à relativiser les résultats des modèles d'impact économique et soulignent la nécessité d'étudier la question des retombées économiques d'un grand chantier sous un angle plus large. Ils mettent en évidence l'enjeu pour les acteurs locaux et les constructeurs de prévoir cette chute d'activité pour sécuriser la trajectoire des entreprises locales et de leurs salariés, afin d'entretenir la dynamique économique de court terme générée par la construction de la ligne. Dans le cadre du projet LGV SEA, des comités départementaux et régionaux associant les acteurs locaux et le constructeur ont en charge de suivre cette baisse d'activité pour favoriser le travail d'orientation, d'insertion et de formation professionnelle de Pôle Emploi.

Tableau 4 : Répartition de l'impact économique par branche d'activité

\begin{tabular}{|c|c|c|c|c|c|}
\hline \multirow{2}{*}{ Branches d'activité } & \multicolumn{4}{|c|}{ Richesse totale $(\mathrm{Y})$} & \multirow{2}{*}{$\begin{array}{l}\text { Indice de } \\
\text { spécialisation } \\
\text { sectorielle }\end{array}$} \\
\hline & Production & Valeur ajoutée & $\begin{array}{l}\text { Emplois } \\
\text { (ETPA) }\end{array}$ & $\begin{array}{c}\text { Emplois } \\
\text { (en \% du total) }\end{array}$ & \\
\hline Agriculture, sylviculture et pêche & $25021311 €$ & $8761007 €$ & 320 & $2.3 \%$ & $2.2 \%$ \\
\hline $\begin{array}{l}\text { Industries extractives, énergie, eau, } \\
\text { gestion des déchets et dépollution }\end{array}$ & $301515250 €$ & $93347024 €$ & 758 & $5.5 \%$ & $2.0 \%$ \\
\hline $\begin{array}{l}\text { Fabrication de denrées alimentaires, } \\
\text { de boissons et de produits à base de } \\
\text { tabac }\end{array}$ & $61076703 €$ & $14691171 €$ & 263 & $1.9 \%$ & $2.8 \%$ \\
\hline Cokéfaction et raffinage & $22044222 €$ & $1002230 €$ & 27 & $0.2 \%$ & $0.0 \%$ \\
\hline $\begin{array}{l}\text { Fabrication d'équipements électriques, } \\
\text { électroniques, informatiques } \\
\text { fabrication de machines }\end{array}$ & $27110466 €$ & $8240364 €$ & 145 & $1.1 \%$ & $2.1 \%$ \\
\hline Fabrication de matériels de transport & $20609470 €$ & $2890546 €$ & 51 & $0.4 \%$ & $1.6 \%$ \\
\hline Fabrication d'autres produits industriels & $446487262 €$ & $146420621 €$ & 2503 & $18.1 \%$ & $7.7 \%$ \\
\hline Construction & $308041781 €$ & $243505173 €$ & 6472 & $46.9 \%$ & $7.4 \%$ \\
\hline $\begin{array}{l}\text { Commerce ; réparation d'automobiles } \\
\text { et de motocycles }\end{array}$ & $47060803 €$ & $25688120 €$ & 544 & $3.9 \%$ & $14.2 \%$ \\
\hline Transports et entreposage & $25299342 €$ & $12695555 €$ & 210 & $1.5 \%$ & $5.7 \%$ \\
\hline Hébergement et restauration & $22521972 €$ & $11669723 €$ & 283 & $2.0 \%$ & $3.6 \%$ \\
\hline Information et communication & $27238085 €$ & $13313043 €$ & 138 & $1.0 \%$ & $1.7 \%$ \\
\hline
\end{tabular}

${ }^{9}$ L'indice de spécialisation sectorielle est un indicateur statistique qui permet de présenter la répartition des emplois selon les différents secteurs d'activité qui composent un tissu économique local. Pour chacun des secteurs, il s'agit de calculer son poids dans l'emploi total du territoire d'étude. 


\begin{tabular}{|l|c|c|c|c|c|}
\hline \multicolumn{1}{|c|}{ Branches d'activité } & \multicolumn{4}{c|}{ Richesse totale (Y) } & Indice de \\
\hline Activités financières et d'assurance & $32620748 €$ & $13736039 €$ & 181 & $1.3 \%$ & $3.5 \%$ \\
\hline Activités immobilières & $69765399 €$ & $57694454 €$ & 53 & $0.4 \%$ & $0.9 \%$ \\
\hline $\begin{array}{l}\text { Activités scientifiques et techniques } \\
\text { services administratifs et de soutien }\end{array}$ & $155897336 €$ & $79492267 €$ & 1390 & $10.1 \%$ & $8.3 \%$ \\
\hline $\begin{array}{l}\text { Administration publique, enseignement, } \\
\text { santé humaine et action sociale }\end{array}$ & $16835368 €$ & $12717956 €$ & 248 & $1.8 \%$ & $32.8 \%$ \\
\hline Autres activités de services & $14524941 €$ & $9231898 €$ & 215 & $1.6 \%$ & $3.4 \%$ \\
\hline Total & $1623670461 €$ & $755097192 €$ & 13799 & $100.0 \%$ & $100.0 \%$ \\
\hline
\end{tabular}

\section{Conclusion}

En nous appuyant sur la littérature dévolue à l'analyse du développement régional, et en mobilisant un modèle d'impact économique Input-Output hybride d'inspiration keynésienne, nous mesurons les bénéfices pour l'activité économique des régions traversées de la construction de la LGV SEA Tours-Bordeaux pour la période 20112013. L'effet régional d'un programme de relance via la construction d'infrastructures de transport est réellement efficace, si et seulement si, le projet contribue à réduire le chômage et qu'il ne détourne pas la main d'œuvre locale des emplois locaux (OCDE, 2002). Dans le cadre du projet SEA, nous observons cette configuration conférant à celui-ci un véritable rôle de relance de l'activité locale. En effet, la croissance du taux de chômage a été ralentie grâce à la construction de la ligne, notamment dans les zones d'emplois proches du tracé. Par ailleurs, une enquête menée auprès de salariés locaux du chantier montre que la majorité d'entre eux (entre $48 \%$ et $70 \%$ selon la catégorie socio-professionnelle) était au chômage avant de travailler sur le projet SEA (Fouqueray, 2014; Fouqueray et Lempereur, 2014).

Les résultats que nous présentons dans cet article sont également le fruit de la coordination et des négociations entre les acteurs publics nationaux et locaux d'une part, et le constructeur et le concessionnaire de la ligne d'autre part quant à la mobilisation du tissu économique local. En effet, sous condition du financement du projet par les collectivités territoriales, des engagements contractuels ont été pris quant à la localisation des soustraitants et des fournisseurs. A défaut de pouvoir présager de ce qu'aurait été l'impact économique de la construction de la LGV SEA pour les régions traversées sans ces engagements contractuels, il nous semble important de souligner que les retombées économiques estimées ne sont pas le fruit d'une coordination par le marché totalement libre.

Par ailleurs, nous démontrons qu'il est possible de recourir, en France, à d'autres méthodes d'évaluation d'impact économique que celle de la base économique, sous condition d'un travail d'enquête conséquent et de la mobilisation appropriée de données nationales. Nous mettons en évidence que la propension des entreprises locales à se fournir et à sous-traiter localement influence grandement la mesure de l'impact économique, tout comme le calcul de l'injection nette ou encore la propension à dépenser localement des salariés directs et indirects. Avec des résultats proches d'études conduites outre-Atlantique, nous soulignons les facteurs clés à l'origine de cet impact. II apparaît que la méthode retenue nous permet d'estimer avec précision l'intensité de l'impact observé à la fois en termes de production, d'emplois et de valeur ajoutée.

Enfin, au-delà des mesures de l'impact économique que nous réalisons dans cet article, nous envisageons de poursuivre l'analyse en estimant la rentabilité sociale pour les collectivités territoriales des régions traversées. Cette estimation nécessitera de prendre en compte l'ensemble des coûts liés à l'accueil d'un grand chantier, notamment ceux liés à la mise en œuvre de politiques publiques de formation professionnelle et d'insertion. $\mathrm{A}$ moyen et long terme, nous chercherons à évaluer s'il existe des effets durables pour les territoires traversés et les agents économiques locaux. Nous nous interrogerons sur la capacité de ces derniers à capitaliser sur le développement économique permis par le chantier. Pour faire ce travail, nous réaliserons des études de trajectoires auprès d'entreprises et de salariés locaux. 


\section{Bibliographie}

Aandréani E., 1967. Le coût d'opportunité. Revue Économique, vol.18, n5, pp. 840-858.

Barget E., Gouguet J.-J., 2010. L'accueil des grands événements sportifs : Quel impact économique ou quelle utilité sociale pour les régions? L'exemple de la coupe du monde de rugby 2007 en France. Région et Développement, vol. 31, pp. 93-118.

Barget E., Gouguet J.-J., 2011. De l'importance des dépenses des spectateurs étrangers dans l'impact touristique des grands événements sportifs. Téoros : Revue de Recherche en Tourisme, vol.30, n², pp. 105-119

Berion P., 2002. La construction d'une grande infrastructure de transport et ses premiers effets territoriaux: le cas de l'autoroute A39, section de Dole à Bourg-en-Bresse. Géocarrefour, vol. 77, n¹, pp. 7-20.

Berion P., Faivre E., Grosjean F., Mathieu D., Petitjean N., 2005. Bilan des observations 1993-2004 4b. L'impact du chantier de l'autoroute A39. Observatoire de l'autoroute A39.

Berion P., Sauter A., 2011. Présentation et mise en perspective des retombées économiques et sociales. Les retombées économiques et sociales du chantier de la LGV Rhin-Rhône

Blake A., 2005. Economic Impact of the London 2012 Olympics.

Bouba-Olga O., 2006. Evaluation de l'impact économique du Parc de Futuroscope: quelques éléments de méthodologie.

Burmeister A., 1997. Un grand chantier d'infrastructure peut-il avoir des effets durables en termes de développement industriel régional ? Le cas de la construction du Tunnel sous la Manche, Infrastructures de Transport et Territoires: Approches de Quelques Grands Projets, Collection Emploi, Industrie et Territoire. L'Harmattan, Paris, pp. 151-181.

Catin M., Nicolini V., 2005. Les effets multiplicateurs des dépenses militaires de la DCN. Toulon sur l'économie varoise. Revue D'Économie Régionale Urbaine, $n^{\circ} 4$, pp. 451-480.

Cohen I., Freiling T., Robinson E., 2012. The Economic Impact and Financing of Infrastructure Spending.

Davezies L., 2008. La République et ses territoires: la circulation invisible des richesses, La République des idées. Seuil, Paris.

Decaluwé B., Martens A., Savard L., 2001. La politique économique du développement et les modèles d'équilibre général calculable: une introduction à l'application de l'analyse mésoéconomique aux pays en développement. Les Presses de l'Université de Montréal.

Fofana I., 2007. Elaborer une matrice de comptabilité sociale pour l'analyse d'impacts des chocs et politiques macroéconomiques.

Fouqueray E., 2014. Les effets induits de la construction de la LGV SEA pour les départements traversés : une analyse intermédiaire des modes de vie et de consommation des cadres et des ETAM. Observatoire socioéconomique de la LGV SEA - LISEA, Poitiers.

Fouqueray E., 2013. Sous-traitance et fourniture du chantier de la LGV SEA Tours-Bordeaux : quelles retombées pour les territoires ? Observatoire socio-économique de la LGV SEA - LISEA, Poitiers.

Garrabé P.M., 2008. La valeur d'activité totale d'une opération de développement local: les multiplicateurs territoriaux : théorie et application.

Green D.I., 1894. Pain-Cost and Opportunity-Cost. The Quarterly Journal of Economics, vol.8, n², pp. 218-229.

Hoyt H., 1954. Homer Hoyt on Development of Economic Base Concept.

Johnson P., Thomas B., 1990. Measuring the Local Employment Impact of a Tourist Attraction: An Empirical Study. Regional Studies, vol. 24, n5, pp. 395-403. 
Lempereur A., Fouqueray E., 2014. Les effets induits de la construction de la LGV SEA pour les départements traversés: une analyse intermédiaire des modes de vie et de consommation des compagnons. Observatoire socio-économique de la LGV SEA - LISEA, Poitiers.

Lynch T., 2000. Analyzing the economic impact of transportation project using RIMS II, IMPLAN AND REMI. Office of Research and Special Programs U. S. Department of Transportation.

Manceau E., Bouhedda A., Ray J.-B., 2012. La Ligne à Grande Vitesse Tours-Bordeaux et ses effets vus par les acteurs du territoire. Observatoire socio-économique de la LGV SEA - LISEA, Poitiers.

McCann P., 2001. Urban and Regional Economics. Oxford University Press.

North D.C., 1955. Location Theory and Regional Economic Growth. Journal of Political Economy, vol. 63, $n^{\circ} 3$, pp. 243-258.

OCDE, 2002. Investissements en infrastructure de transport et développement régional, Paris.

Petitjean N., 2003. L'impact socio-économique des chantiers de grandes infrastructures de transport. Thèse de doctorat.

Pratt R.T., 1968. An Appraisal of the Minimum-Requirements Technique. Economic Geography, vol. 44, n², pp. 117-124.

Réseau Ferré de France, 2010. Les retombées économiques et sociales du chantier, Les cahiers de la LGV Rhin-Rhône.

Richardson H.W., 1985. Input-Output and Economic Base Multipliers: Looking Backward and Forward. Journal of Regional Science, vol. 25, n4, pp. 607-661.

Segessemann A., Crevoisier O., 2013. The circulation of wealth beyond economic base theory : alternatives to productive economics? The role of the residential economy in attracting income to switzerland. Maison d'Analyse des Processus Sociaux.

Sombart W., 1902. Der moderne Kapitalismus. Duncker \& Humblot, Leipzig.

Stringer Y., 1992. Le mirage des retombées économiques. École des hautes études commerciales, Centre d'études en administration internationale, Montréal.

Talandier M., 2012. Géographie et impacts socioéconomiques des migrations d'agrément dans les espaces ruraux français. Migrations d'agrément : du tourisme à l'habiter. pp. 181-205.

Taroux J.-P., 1988. Rapport méthodologique sur le modèle IMPACT. Observatoire Économique et Statistique des Transports, Paris.

Tiebout C.M., 1956. A Pure Theory of Local Expenditures. Journal of Political Economy, vol. 64, n5, pp. 416424.

Ullman E.L. Dacey, M.F., 1960. The Minimum Requirements Approach to the Urban Economic Base. Papers in Regional Science, vol. 6, n¹, pp. 175-194.

Vollet D., 2014. Analyse de l'impact économique de la chasse sur l'emploi local en France : Quelles perspectives pour un développement territorial durable des activités de loisirs? Téoros : Revue de Recherche en Tourisme, vol. 32, n¹, pp. 56-68.

Vollet D., Brétière G., 2008. La chasse : Quel impact économique sur l'emploi local ? Une première approche à partir de quelques études de cas. Présenté au colloque SFER "Chasse, Territoires et Développement durable Outils d'analyse, enjeux et perspectives" Clermont-Ferrand, France.

Vollet D., Dion Y., 2001. Les apports potentiels des modèles de la base économique pour guider la décision publique. Revue d'Économie Régionale et Urbaine, n², pp. 179-196.

Wubneh M., 2008. US Highway 17 and its impact on the economy of eastern North Carolina. Urban \& Regional Planning Program Department of Geography East Carolina University. 